\title{
Le chemin de l'espoir
}

\section{Alain Decaux}

\section{ADRESSE}

Alain Decaux : de l'Académie française, Ministre délégué auprès du Ministre d'Etat, Ministre des Affaires étrangères, chargé de la Francophonie. Ministère des Affaires étrangères, 7, rue de Talleyrand, 75700 Paris, France.

$\mathrm{m} / \mathrm{s} n^{\circ} 3 \mathrm{vol} .6$, mars 90

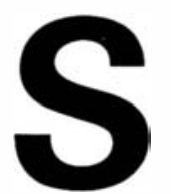

i pour des êtres humains, les anniversaires sont entachés chaque année d'un peu plus de nostalgie, pour un journal ils témoignent d'une vitalité que la pérennité vient confirmer. Ainsi médecine/sciences est en bonne santé : ce cinquantième numéro prouve qu'il a su, par la qualité de ses contenus, conquérir un public exigeant et qu'il parvient à un stade de maturité et d'autonomie reconnues.

Je cite souvent médecine/sciences comme un bel exemple de démarche volontaire et rationnelle, reposant sur une analyse précise d'un secteur donné. Le succès de l'entreprise montre que, pour l'usage du français dans les sciences et dans les techniques, une autre dynamique que celle de la résignation est possible.

Les quelque deux mille personnalités du monde scientifique qui, répondant à mon invitation, sont venues participer les 9 et 10 janvier derniers, au Forum de la communication scientifique et technique, ont insisté sur ce problème essentiel des publications. Si nous ne donnons pas l'exemple, si nous nous soumettons passivement au diktat des grandes revues angloaméricaines, si nous considérons le fait de nous exprimer dans une langue étrangère comme un signe de distinction, alors la communauté scientifique francophone que nous nous efforçons de construire a bien peu de chances de survie.

En ce sens, l'existence de bonnes revues de synthèse, comme de manuels pour l'enseignement supérieur, est indispensable à la " maintenance " de cette communauté qui a besoin de s'informer rapidement et régulièrement, de suivre l'évolution des recherches et des progrès - pour sa pratique professionnelle comme pour la formation de ceux qui seront appelés à prendre demain la relève. Produit exemplaire de la coopération entre le Québec et la France, médecine/sciences montre que les médecins et les biologistes francophones peuvent s'exprimer dans leur langue sans être ipso facto marginalisés au sein de la communauté scientifique internationale.

Merci à médecine/sciences de nous l'avoir rappelé. Merci à toutes celles et à tous ceux qui ont conçu cette revue et qui, avec un soin attentif, ont veillé à sa croissance. Merci à l'équipe de médecine/sciences d'avoir montré à la Francophonie scientifique le chemin de l'espoir

\section{TIRÉS A PART}

A. Decaux. 